\title{
WERTTRANSFER UND UNTERENTWICKLUNG -
}

\section{Bemerkungen zu Aspekten der neueren Diskussion um Weltmarkt, Unterentwicklung und Akkumulation des Kapitals in unterentwickelten Ländern (anhand von E. Mandel, Der Spätkapitalismus (1))}

In der neueren marxistischen Diskussion (2) um Imperialismus und Unterentwicklung hat der Begriff des „Werttransfers" einen zentralen Stellenwert. Anhand einer - behaupteten - permanenten Wertübertragung von den Ländern der Dritten Welt in die kapitalistischen Industrienationen, die ihre Ursache im unterschiedlichen Stand der Entwicklung der Produktivkraft der Arbeit hat, kommen die Teilnehmer an dieser Diskussion - bei aller unterschiedlicher Vorgehensweise und Differenzen in Detailfragen - stets zu zwei wesentlichen Schlußfolgerungen:

1. Die Entwicklung in den kapitalistischen Industrienationen war und wird weiterhin durch die unterentwickelten Länder bezahlt.

2. Die Unterentwicklung in den Ländern der Dritten Welt ist bleibendes Strukturmoment des kapitalistischen Weltmarkts; eine Entwicklung unter kapitalistischen Vorzeichen ist nicht oder doch nur im begrenzten Ausmaß möglich.

Diese Thesen, die schwerwiegende politische Konsequenzen einschließen, basieren unserer Ansicht nach allerdings auf theoretischen Überlegungen, die in Teilen falsch oder aber doch zumindest zu undifferenziert und unklar sind, um entsprechende Aussagen treffen zu können. (3) Im folgenden soll, unter Fortführung vorangegangener Diskussionen, zunächst in den Abschnitten 1 und 2 - ausgehend vom Wertbildungsprozeß - das Problem des Extramehrwerts und der internationalen Werte diskutiert werden, um dann im dritten Abschnitt die Wirkung des ungleichen Tauschs auf den Akkumulationsproze $\beta$ des Kapitals in der Dritten Welt und in den Industrienationen zu untersuchen.

1) E. Mandel, Der Spätkapitalis mus, Irankfurt 1972. An dieser Stelle soll anhand der eineinschlägigen Thesen in diesem Buch lediglich die Diskussion mit Mandel zu diesem Thema fortgefüht werden; eine Auseinandersetzung mit Mandel in Form einer Rezension ist zu cinem späteren Zeitpunkt beabsichtigt.

2) Vgl. dazu S. Amin, L'Acculumation a l' Echelle mondiale, Paris 1970; Ch. Bettelheim, Vorwort und Kommentar, in: A. Emmanuel, L' Echange Inegal, Paris 1969; A. Lmmanuel, a. a. O., Chr. Palloix, Problemes de la croissance en economic ouverte, Paris 1969 ,

3) A. G. Frank, Kapitalismus und Unterentwicklung in Lateinamerika, Frankfurt 1969. Dem steht zumindest die Annahme von Marx gegenüber, das das Kapital über dic Herstellung des Weltmarkts uitu dic Ausbeutung der Kolonien gerade diese unteren twickelten Gebicte entwickeln werde. Vgl. z. B. Karl Marx, Dic künftigen Ergebnisse der britischen Herrschaft in Indien, in: Marx-Engels-Werke, Bd. 9, Berlin (DDR) 1960, S. 220 ff. 


\section{Ernest Mandel}

\section{DER STURZ DES DOLLARS}

\section{Eine Marxistische Analyse der Weltwährungskrise}

Kaum ein Thema machte in den Ictzten Jahren soviel Schlagzeilen, wie das der Währungskrisen und der Inflation. Wïhrend Arbeiter um Teuerungszulagen kämpfen und eine Krise des Weltwährungssystems aut dic andere folgt, begnügt sich dic ökonomische Theorie häufig damit, die Währungskrisen nur begrifflich abzuleiten. Eines der zentralen Themen kapitalistischer Wirklichkeit gerät so in Gefahr, eine Sache für Spezialisten zu werden und den Bezug zur konkreten Realität zu verlieren.

Dieses Buch schafft Abhilte, befreit die Fragen der Währungskrisen und Inflation durch seine leicht verständliche Form von ihrer spezialistischen Unverständlichkeit.

Mandel geht in seiner Analyse von der konkreten Entwicklung der wichtigsten kapitalistischen Länder seit 1964 aus. Die Probleme der Internationalisierung der kapitalistischen Produktion. der multinationalen Konzerne bei weiter bestehender Nationalstaatlichkeit; der Verschärfung der Konkurrenz zwischen den Kapitalien, deren politischer Ausdruck der Protektionismus bei fortgeschrittener internationaler Arbeitsteilung; des Widerspruchs der Funktion des Geldes ats Zirkulationsmittel für den Weltmarkt und als Instrumentarium nationalen Krisenmanagements - all das wird einer eingehenden Behandlung unterzogen. Schließlich ist die Währungskrise für Mandel kcin rein ökonomisches Problem. Vietnamkrieg und Mai 68 sind Ereignisse, deren Auswirkungen auf die Währungskrisen von größter Bedeutung sind.

Der Band ist in vier Teile untergliedert. Ein Abschnitt besteht aus einer Reihe von Aufsätzen, die konkret zu sämtlichen wichtigen währungspolitischen Entscheidungen seit der Pfundkrise von 1964 bis zum August 1973 Stellung beziehen. Ein längerer Beitrag gibt eine geschlossene theoretische Erklärung des gesamten Komplexes und beleuchtet zudem die diversen Reformvorschläge für das zerrüttete Weltwährungssystem. Der dritte Abschnitt besteht aus einem Vorwort, das die Geschichte des Weltwählrungssystems seit Bretton Woods zum Inhalt hat, und einer Einleitung von Ernest Mandel. In dieser Einleitung gibt er eine Zusammenfassung seiner wichtigsten Forschungsergebnisse zur Theorie des Spätkapitalismus und bettet die vorliegenden Aufsätze in diese Untersuchung ein, so daß der Währungsbereich in die Totalität der kapitalistischen Produktionsweise integriert ist. Der vierte Teil dient zur Erleichterung der Arbeit mit dem Text. Er besteht aus einer Chronologie der wichtigsten Ereignisse, die seit 1944 bis jetzt den monetären Sektor betreffen, und aus einem Glossarium, in dem kurz die wichtigsten Begriffe der Analyse noch einmal mit Erläuterungen zusammengestellt sind.

\section{ep 5, 160 Seiten, DM 9.}

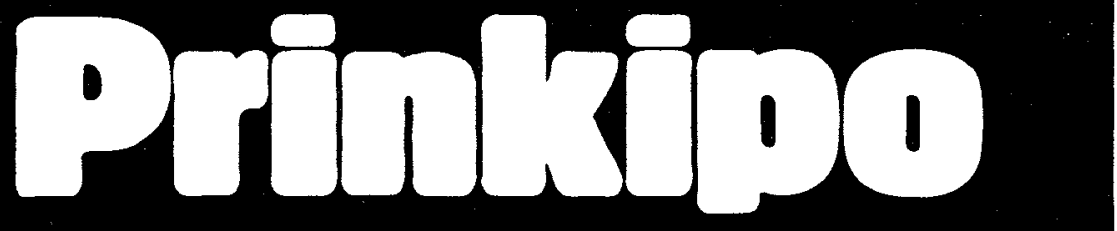

Bei Bestellungen an den Verlag plus DM 1.- Porto, bei Gruppenbestellungen ab 10 Ex. 10\% Rabatt, Lieferung erst nach Eingang der entsprechenden Summe auf unserem Konto. Uber weitere Titel der Reihe EDIfION PRINKIPO und KRITISCHE BIBLIOTHEK DER ARBEITERBEWEGUNG, wie der Zeitschrift KRITIK DER POLITISCHEN OKKONOMIE informiert unser Prospekt, der angefordert werden kann (bitte 40 pfg. Briefmarken beilegen).

Verlag Olle \& Wolter, 1 Berlin 30, Postfach 4310, Postscheckkto. BInW 47006-104, Sparkasse Berlin West, Kto.Nr. 067001142 


\section{Gesellschaftlich notwendige Arbeit, Extramehrwert, Produktionspreis und Wert- transfer}

Eine detaillierte Auseinandersetzung mit Mandels Theorie der internationalen Werte und der damit zusammenhängenden Ableitung der Unterentwicklung setzt zunächst eine kritische Betrachtung seiner Interpretation des Extramehrwerts bei Marx und der Möglichkeit der Wertübertragung zwischen und nicht innerhalb der Branchen voraus. Aus diesem Grunde soll im folgenden zunächst der systematische und kategoriale Zusammenhang bei Marx zu diesen hier angesprochenen Problemen entfaltet werden.

\subsection{Die Noppeltbestimmtheit der gesellschaftlich notwendigen Arbeit}

In der warenproduzierenden Gesellschaft nehmen die Produkte die Form des Werts an; in der Wertform drücken sich - sachlich verhüllt - die gesellschaftlichen Beziehungen von Privatproduzenten aus. Die gesellschaftliche Arbeit wird nicht unmittelbar als solche verausgabt, sondern in der Form der Privatarbeit geleistet. Erst im Austausch erhält diese Privatarbeit ihren gesellschaftlichen Charakter, und zwar in dem Sinne, daß sich erst im nachhinein durch den Austausch erweist, wieviel von der privat verausgabten Arbeit überhaupt gesellschaftlich anerkannt wird - als gesellschaftlich notwendige Arbeit sich darstellt - und wieviel Arbeit in privater Form gesellschaftlich überflüssig war. Nur der Teil der privat verausgabten Arbeit, der sich gesellschaftlich bewährt, konstituiert überhaupt den gesellschaftlichen Wert der Waren. Diese ,gesellschaftlich notwendige Arbeit" bestimmt sich aber nach Marx in doppelter Weise:

„Gesellschaftlich notwendige Arbeitszeit ist Arbeitszeit erheischt, um irgendeinen Gebrauchswert mit den vorhandenen gesellschaftlich normalen Produktionsbedingungen und dem gesellschaftlichen Durchschnittsgrad an Geschick und Intensität der Arbeit herzustellen“ (6) und: „Es ist ... nur das Quantum gesellschaftlich notwendiger Arbeit oder die zur Herstellung eines Gebrauchswerts gesellschaftlich notwendige Arbeit, welche seine Wertgröße bestimmt." (7) Trifft diese Argumentation die technischen Durchschnittsbedingungen (und deren Veränderungen mit der Entwicklung der Produktivkraft der Arbeit, was unten für unsere Argumentation zentralen Stellenwert haben wird), so ist zum anderen gesellschaftlich notwendige Arbeit die Arbeit, die gemäß den auf dem Markt (in der Form der zahlungsfähigen Nachfrage) auftretenden gesellschaftlichen Bedürfnissen auf die verschiedenen Produktionszweige verteilt ist: „Gesetzt endlich, jedes auf dem Markt vorhandene Stïck Leinwand enthalte nur gesellschaftlich notwendige Arbeitszeit. Trotzdem kann die Gesamtsumme dieser Stücke überflüssig verausgabte Arbeitszeit enthalten. Vermag der Marktmagen das Gesamtquantum Leinwand zum Normalpreis von 2 sh per Elle nicht zu absorbieren, so beweist das, daß ein zu großer Teil der gesellschaftlichen Gesamtarbeitszeit in der Form der Leineweberei verausgabt wurde. Die Wirkung ist dieselbe, als hätte jeder einzelne Leineweber mehr als die ge-

4)5) Entfallen

6) Karl Marx, Das Kapital, Bd. 1, Berlin (DDR) 1962, S. 52 (in der Folge zit.: Kapital I)

7) Kapital I, S. 54. 
sellschaftlich notwendige Arbeitszeit aus sein individuelles Produkt verwandt." (8) Die Notwendigkeit der Verteilung der gesellschaftlichen Arbeit auf die verschiedenen Produktionszweige gemäß den gesellschaftlichen Bedürfnissen und deren Einfluß auf die Konstituierung des gesellschaftlichen Werts der Waren wird an anderer Stelle bei Marx noch deutlicher: „Wie es Bedingung für die Waren, daß sie zu ihrem Wert verkauft werden, daß nur die gesellschaftlich notwendige Arbeitszeit in ihnen enthalten, so für eine ganze Produktionssphäre des Kapitals, daß von der Gesamtarbeitszeit der Gesellschaft nur der notwendige Teil auf diese besondre Sphäre verwandt sei, nur die Arbeitszeit, die zur Befriedigung des gesellschaftlichen Bedürfnisses (demand) erheischt. Wenn mehr, so mag jede einzelne Ware nur die notwendige Arbeitszeit enthalten; die Summe enthält mehr als die gesellschaftlich notwendige Arbeitszeit, ganz wie die einzelne Ware zwar Gebrauchswert hat, die Summe aber unter den gegebenen Voraussetzungen einen Teil ihres Gebrauchswerts verliert.“ (9) Diese zweite, das Gebrauchsquantum der Ware berücksichtigende Bestimmung „gesellschaftlich notwendiger Arbeitszeit" wird auch von Engels in einer Kritik an Rodbertus hervorgehoben, wenn er darin beide Bestimmungsmomente zusammenfaßt: „Hätte er (Rodbertus) untersucht, wodurch und wie die Arbeit Wert schafft und daher auch bestimmt und mißt, so käme (i. Original: kann) er auf die gesellschaftlich notwendige Arbeitszeit - notwendig, für das einzelne Produkt sowohl gegenüber anderen Produkten derselben Art, wie auch gegenüber dem gesellschaftlichen Gesamtbedarf." (10)

Zusammenfassend muß also festgehalten werden, daß nur die Verausgabung von Arbeit unter den gesellschaftlichen Durchschnittsbedingungen wertbestimmend wirkt wie andererseits die der kapitalistischen Produktionsweise entsprechende naturwüchsige Form der Verteilung der Arbeit unter die verschiedenen Produktionssphären (die erst über den Austausch, der Realisierung anzeigt, welcher Teil der verausgabten Arbeit gesellschaftlich notwendig, welcher überflüssig war) den Wert der Ware konstituiert. Dabei soll hier schon auf einen Aspekt hingewiesen werden, der in den entsprechenden theoretischen Auseinandersetzungen häufig vernachlässigt wird: Die Entfaltung der Produktivkraft der Arbeit reduziert das gesellschaftlich notwendige Quantum Arbeit pro Ware mittels Arbeitsteilung, Kooperation, Maschinerie und sonstiger beschleunigter Entwicklung der Produktivkräfte und vermindert damit die Reproduktionskosten der Ware (ausgedrückt als notwendiges Quantum Arbeit). (Die äußere Form des immanenten Warenwerts als Produktionspreis und seiner Elemente - konstantes Kapital, Arbeitslohn, Profit etc. - braucht hier nicht weiter betrachtet zu werden; die Bewegung des Produktionspreises und seiner Elemente muß jedoch der o. a. Tendenz, die mit der Entwicklung der Produktivkraft der Arbeit gegeben ist, entsprechen!) Die Konsequenzen der Einbeziehung dieses Aspektes der Wertbestimmung sollen weiter unten aufgezeigt werden.

8) Ebd., S. 121 f.; vgl. auch ebd., S. 122 (Fußnote): ,Und in der Tat ist der Wert der individuellen Elle ja auch nur die Materiatur eines Teils des im Gesamtquantum der Ellen verausgabten gesellschaftlichen Arbeitsquanten."

9) Karl Marx, Theorien über den Mehrwert, Zweiter Teil, Berlin (DDR) 1967, (MEW 26,2), S. 521 .

10) Friedrich Engels, zit. nach Roman Rosdolsky, Zur Entstehungsgeschichte des Marxschen Kapitals, Bd. 1, Frankfurt a. M. 1968, S. 117. 
Nach der in doppelter Weise vorgenommenen Bestimmung der gesellschaftlich notwendigen Arbeitszeit (und der Bedeutung der Entwicklung der Produktivkraft der Arbeit darin), soll nun - aufbauend auf dem 10. Kapitel des dritten Bandes des „Kapitals“ - das Problem des Marktwerts und Marktpreises in unserem Zusammenhang diskutiert werden. Generell wird der Marktwert, der gesellschaftliche Wert der Waren gegenüber den Einzelproduzenten, von den Produktionsbedingungen bestimmt, die innerhalb einer Branche das überwiegende Quantum des gesellschaftlichen Bedürfnisses nach Waren dieser Branche befriedigen. Marx unterscheidet dabei drei Produktionsbedingungen innerhalb einer Branche: die mittleren (Fall I), die schlechtesten (Fall II) und die besten Bedingungen (Fall III) der Produktion. Weichen Angebot und Nachfrage voneinander ab, so pendeln die Marktpreise um die Marktwerte, in extremen Fällen wird dann das Hauptquantum von Waren unter den besten bzw. den schlechtesten Bedingungen produziert. $D$. h. bei langfristigem Ungleichgewicht verschiebt sich das Zentrum (der Marktwert), um das erneut die Marktpreise oszillieren. Übersteigt also die Nachfrage erheblich das Angebot, dann wird der Fall eintreten, daß die Hauptmasse des gesellschaftlichen Bedürfnisses nach Waren aus dieser Branche von den zu den schlechtesten Bedingungen produzierenden Kapitalen mit befriedigt wird. Die mittleren und besten Produktionsbedingungen (Fall I und III) sind dann ihrer Kapazität nach nicht in der Lage, der vorhandenen Nachfrage voll zu entsprechen, weshalb die Produzenten mit den schlechtesten Produktionsbedingungen auf dem Markt erscheinen können. Umgekehrt, umgekehrt!

Bei kurzfristigem Auseinanderfallen von Angebot und Nachfrage bleibt also das Zentrum der Marktpreise, der Marktwert, erhalten, um das die Marktpreise pendeln. Weichen Angebot und Nachfrage über eine längere Zeit extrem voneinander $a b$, so verschiebt sich das Gravitationszentrum hin zu den besten bzw. schlechtesten Bedingungen (infolge der veränderten - verschlechterten bzw. verbesserten - Reproduktionsbedingungen der Waren). Insofern ist also auch nicht die $\mathrm{Nach}$ frage wertbestimmend, sondern es sind die veränderten Reproduktionsbedingungen der Waren, die die Wertveränderungen hervorrufen! (11) Hier sei noch bemerkt, daß es in diesem Zusammenhang falsch ist, von der Vorstellung eines gewogenen arithmetischen Mittels auszugehen (12), da bei dieser Berechnungsweise jene geleistete Arbeitszeit mit in die Berechnung eingeht, die aufgrund der (von uns oben entwikkelten) Bestimmungen der gesellschaftlich notwendigen Arbeit nicht wertbildend sein kann (davon abgesehen, daß der Marktwert ohnehin nicht empirisch erfaßbar ist; Wert existiert überhaupt empirisch faßbar nur im Geldausdruck, in dem der Wertbildungsprozeß zum Abschluß gekommen ist!).

11) Karl Marx, Das Kapital, Bd. 3, Berlin (DDR) 1964, (in der Folge zit. als Kapital III), S. 150: „Der Wert jeder Ware - also auch der Waren, aus denen das Kapital besteht ist bedingt, nicht durch die in ihr selbst enthaltene, notwendige Arbeitszeit, sondern durch die gesellschaftlich notwendige Arbeitszeit, die zu ihrer Reproduktion erheischt ist. Diese Reproduktion kann erfolgen unter erschwerenden oder unter erleichternden Umständen, verschieden nach den Bedingungen der ursprünglichen Produktion."

12) Vgl. dazu Gunther Kohlmey, Karl Marx* Theoric von den internationalen Werten, in: Probleme der Politischen Ökonomie (Jahrbuch), Bd. 5, Berlin (DDR) 1962, S. 44 ff., und die $\Lambda$ uscinandersetzung mit Kohlmey, in: Buseh/Schöller/Seclow, Weltmarkt und Weltwährungskrise, Bremen 1971, S. 25 f. Auf dic darin enthaltenen Thesen wird weiter unten cingegangen. 
Diese Verschiebung des Gravitationszentrums infolge der langfristig veränderten Verhältnisse von Angebot und Nachfrage bedeutet also, daß sich die Reproduktionskosten der Waren verändern, nicht jedoch, daß Nachfrage oder Angebot selbst wertbestimmend sind. Übersteigt nämlich z. B. die gesellschaftliche Nachfrage das Angebot in der Branche langfristig, so wird demnach eine größere Masse der betreffenden Waren zu schlechteren Produktionsbedingungen hergestellt, d. h. die Reproduktionskosten der Waren erhöhen sich allgemein bzw. die zur Herstellung einer Ware gesellschaftlich notwendige Arbeitszeit nimmt zu. Auf der Basis dieser Überlegungen soll nun das Problem des Extramehrwerts (auf das sich die bisherige Diskussion zentral bezog (13)) behandelt werden.

\subsection{Extramehrwert, Durchschnittsprofit und Werttransfer}

Marx führt den Extramehrwert auf die im Vergleich zu den Konkurrenten außerordentliche Produktivkraft in einem Unternehmen zurück. Der Kapitalist, der über solche besonderen Produktionsbedingungen verfugt, produziert deshalb in derselben Zeit mehr Produkte als in der Branche üblich, weil er weniger Arbeit als in dieser Branche üblich zur Herstellung eines Produkts anwenden muß bzw. die Produktion einer Ware kostet ihn weniger als seinen Konkurrenten in derselben Branche. Die Waren in der Branche werden zu ihrem gesellschaftlichen Wert, Marktwert (bzw. Marktpreis), verkauft, der jeweils gesellschaftlich notwendige Arbeit repräsentiert. Da jedoch der Kapitalist, der über eine außerordentliche Produktivkraft der Arbeit verfügt, die Waren zu unterdurchschnittlichen Kosten herstellen kann, also weniger Arbeit in die Produktion dieser Waren eingeht, spricht Marx davon, daß die unter diesen Bedingungen angewandte Arbeit als potenzierte Arbeit wirkt. (14) Denn der individuelle Wert dieser Waren ist niedriger als der gesellschaftliche Wert der Waren der gleichen Art aus dieser Branche, da in dem begünstigten Unternehmen der für die Arbeitskraft verauslagte Kapitalteil schneller reproduziert wird als allgemein in der betreffenden Branche üblich. Das Äquivalent für den Arbeitslohn wird somit in kürzerer Zeit reproduziert, was eine Erhöhung des angeeigneten Mehrwerts für das bestimmte Einzelkapital einschließt. Die Möglichkeit der Produktion eines solchen Extramehrwerts besteht so lange, wie diese außerordentlichen Produktionsbedingungen sich nicht innerhalb der Branche verallgemeinert haben.

Der Begriff des, individuellen Werts“"im Zusammenhang mit dem Extramehrwert bei Marx kann allerdings nicht heißen, daß es wirklich einen individuellen Wert gäbe. Wenn Marx vom individuellen Wert spricht, so drïckt er damit lediglich die Gegensätzlichkeit von privat verausgabter und gesellschaftlich notwendiger Arbeit aus. Daß der individuelle Wert einer Ware nicht als Ausgangspunkt der Bestim-

13) Vgl. dazu Busch/Schöller/Seelow, a. a. O., Kohlmey, a. a. O., Chr. Neusüß, Imperialismus und Weltmarktbewegung des Kapitals, Erlangen 1972, bes. S. 125 ff.; Mandel, a. a. O. und ders., Marxistische Wirtschaftstheorie, Frankfurt a. M. 1968.

14) Vgl. dazu Kapital I, S. 337: ,Die Arbeit von ausnahmsweiser Produktivkraft wirkt als potenzierte Arbeit oder schafft in gleichen Zeiträumen höhere Werte als die gesellschaftliche Durehschnittsarbeit derselben Art ... Der Kapitalist, der die verbesserte Produktionswe ise anwendet, eignet sich daher einen größern Teil des Arbeitstages für die Mehrarbeit an, als die übrigen Kapitalisten in demselben Geschäft. Er tut im einzelnen, was das Kapital bei der Produktion des relativen Mehrwerts im grolsen und ganzen tut. Andrerseits aber verschwindet jener Extramehrwert, sobald die neue Produktionsweise sich verallgemeinert und damit die Differenz zwischen dem individuellen Wert der wohlfeiler produzierten Waren und ihrem gesellschaftlichem Wert verschwindet." 
mung des gesellschaftlichen Werts genommen werden darf, kommt in folgender Passage deutlich zum Ausdruck: ,Der wirkliche Wert einer Ware ist aber nicht ihr individueller, sondern ihr gesellschaftlicher Wert, d. h. er wird nicht durch die $\mathrm{Ar}$ beitszeit gemessen, die sie im einzelnen Fall dem Produzenten tatsächlich kostet, sondern durch die gesellschaftlich zu ihrer Produktion erheischte Arbeitszeit." (15) Ist diese hier vorgenommene Interpretation von gesellschaftlich notwendiger Arbeit und Extramehrwert richtig, so folgt darauf, daß es innerhalb einer Branche zu keiner Umverteilung von Werten kommen kann. Die Arbeit, die unter Produktionsbedingungen verausgabt wird, die unter den in der betreffenden Branche geltenden massenhaften Produktionsbedingungen liegen, geht also verloren, ist nicht wertbildend.

Mandel nimmt demgegenüber an, daß auch der Extramehrwert innerhalb einer Branche und somit jeder Surplusprofit durch einen Werttransfer zustande kommt. Innerhalb einer Branche gibt es jedoch, wie wir zeigen konnten, keine Wertübertragung! Die gesamte Wertschöpfung in einer Branche bleibt auch bei Vorhandensein eines Extramehrwerts unverändert. Durch diesen Extramehrwert wird kein zusätzlicher Wert geschaffen, sondern der produktivere Kapitalist erzielt lediglich - vermittelt über die vorhandenen ausnahmsweisen Produktionsbedingungen in seinem Unternehmen - einen höheren Profit auf sein angelegtes Kapital gegenüber dem Durchschnitt in der Branche. Der Extramehrwert ist also keineswegs so zu fassen, daß die Produzenten, die unter den in der Branche durchschnittlichen Produktionsbedingungen produzieren, einen Mehrwert schaffen, der zu den Kapitalen, die zu den besten Produktionsbedingungen produzieren, ,transferiert" wird und dort den Extramehrwert bildet (wie von Mandel angenommen). Mandel unterscheidet bei Betrachtung des Surplusprofits nicht klar zwischen der Konkurrenz innerhalb der Branche und jener zwischen den Branchen. Zumindest an der folgenden Passage läßt sich jedoch zeigen, daß M. ausdrücklich von Extramehrwert als Resultat des Werttransfers innerhalb der Branche spricht: ,Wenn Marx sagt, daß die unter der durchschnittlichen Arbeitsproduktivität arbeitenden Betriebe weniger als den Durchschnittsprofit erhalten, und daß dies letztenendes der der Tatsache entspricht, daß sie gesellschaftliche Arbeit vergeudet haben, so bedeutet diese Formel nur, daß der von ihren Arbeitern tatsächlich produzierte Wert bzw. Mehrwert auf dem Markt durch besser arbeitende Firmen angeeignet wird, keineswegs, daß dort weniger Wert und Mehrwertschöpfung, als durch die geleisteten Arbeitsstunden angezeigt wird, stattgefunden hat." (16) Noch deutlicher wird diese u. E. falsche Auffassung vom Werttransfer innerhalb einer Branche in dem folgenden Zitat: „Dann ist also - eine identische Mehrwertrate vorausgesetzt - die gesamte in diesem Produktionszweig erzeugte Mehrwertmasse gleich der gesamten Profitmasse. Und dann kann der Surplusprofit der mit überdurchschnittlicher Arbeitsproduktivität arbeitenden Firmen nur aus einem Werttransfer auf Kosten der mit unterdurchschnittlicher Arbeitsproduktivität arbeitenden Firmen erklärt werden." (17) (Die übrigens in diesem Fall von Mandel angenommene unmittelbare Identität von Mehrwertmasse und Profitmasse innerhalb einer Produktionssphäre ist falsch und steht auch im Widerspruch zu seiner weiteren Ableitung eines Surplusprofits aus dem Werttransfer zwischen den Branchen bei Herstellung der Durchschnittsprofitrate und der Produktionspreise, bei der ja gerade die Nichtidentität von produziertem Mehrwert

15) Kapita1 III, S. 336.

16) Mandel, Der Spätkapitalismus, a. a. O., S. 94.

17) Ebd., S.95. 
und angeeignetem Profit gese tzt ist. Vgl. dazu unten!)

Ist so einerseits Mandels Interpretation des Extramehrwerts zurückzuweisen, da es innerhalb der Branche zu keiner Wertiibertragung kommen kann, so muß ihm insofern zugestimmt werden (und die früher eingenommene kritische Position zurückgenommen werden (18), wenn er eine „Wertübertragung" zwischen den Branchen konstatiert. Dies soll im folgenden genauer ausgeführt werden.

Zunächst kann gesagt werden, daß sich unter normalen Bedingungen zwischen den Branchen infolge des Kapitalflusses (dessen ungehinderte Bewegung hier vorausgesetzt werden muß!) eine durchschnittliche Profitrate herstellt. Denn der überdurchschnittliche Branchenprofit provoziert den Abfluß der Kapitale aus den Branchen mit niedrigerer durchschnittlicher Profitrate bzw. veranlaßt die in der profitableren Branche tätigen Kapitale weiterhin in dieser Branche zu akkumulieren. Über die Kapitalwanderung aus den Branchen mit unterdurchschnittlicher und durchschnittlicher Profitrate ergibt sich im Zwang der Konkurrenz die Tendenz zu den Branchen mit überdurchschnittlicher Profitrate und damit zur Angleichung der unterschiedlichen Branchenprofitraten hin zu einer gesellschaftlichen Durchschnittsprofitrate. Diese ,gesellschaftliche Durchschnittsprofitrate“, die nie real erreicht wird, sondern nur der Tendenz nach über den Fluß der Kapitale und die damit erfolgende Nivellierung der Branchenprofite zustandekommt, führt dazu, daß die Kapitale im Durchschnitt anteilmäßig gleichen Profit entsprechend der Größe der einzelnen Kapitale auf sich vereinigen, d. h. daß sich Produktionspreise herausbilden. Da nun aber gesamtgesellschaftlich die Summe der Profite gleich sein muß mit der gesamten Mehrwertmasse, muß es im Ergebnis zu einer Umverteilung von Wertbestandteilen gekormmen sein. Denn die erzielten Profite einer Branche können nicht identisch sein mit dem Quantum Mehrwert, das diese Branche zur gesamten gesellschaftlichen Mehrwertmasse beigetragen hat. Gesamtgesellschaftlich besteht ein Mehrprodukt und damit in der kapitalistischen Gesellschaft ein entsprechendes Quantum Mehrwert, das sich im Profit darstellt, wobei der Beitrag der einzelnen Branchen gemäß der organischen Zusammensetzung der in den verschiedenen Branchen angelegten Kapitale verschieden ist (entsprechend der Anzahl der angewandten Arbeitskräfte!). Theoretisch besteht hier die Schwierigkeit, die Profitmasse der einzelnen Branchen mit der gesamtgesellschaftlichen Mehrwertmasse in ihrem Zustandekommen zu vermitteln. Insofern allerdings die Summe der Profite der gesellschaftlichen Mehrwertmasse entsprechen muß, muß es im Ergebnis - wie schon dargestellt - zu einer Umverteilung gekommen sein, auch wenn der Profit des Einzelkapitals nichts mit dem im einzelnen produzierten Mehrwert zu tun hat! (19) Jedoch kann im weiteren in unserem Zusammenhang die Diskussion über die Vermittlung von produziertem Mehrwert und angeeignetem Profit (für die Produktionssphäre als Ganzes) vernachlässigt werden, da sie für die Ableitung der Unterentwicklung und die Frage der Bildung der internationalen Werte nicht von Bedeutung ist.

In unserem Zusammenhang sind die bisherigen Ausführungen deshalb wichtig, weil Mandel über einen behaupteten Werttransfer auf dem Weltmarkt (in der Form des „ungleichen Tauschs") die Unterentwicklung zu erklären versucht. Dabei geht

18) Vgl. Busch/Schöller/Seelow, a. a. O., S. $21 \mathrm{ff}$.

19) Mandel, der sich auf S. 89 ff. mit der Kritik an seiner Vorstellung vom Werttransfer auseinandersetzt (vgl dazu Fußnote 18), in der jeder Werttransfer - auch solcher 7 wischen den Branchen - abgelehnt wird, ist deshalb zuzustimmen, soweit sich die ,Umvertcilung" der Wertbestandteile im oben entwickelten Sinn auf die Konkurrenz zwischen den Branchen bezieht! 
er in seiner Ableitung der Unterentwicklung von der Annahme aus, daß es auf dem Weltmarkt (noch) keine Durchschnittsprofitrate gibt, daß es jedoch demgegenüber zur Bildung internationaler Werte kommt. Wenn oben gezeigt wurde, daß seine Ableitung des Extramehrwerts falsch ist, so wird diese Kritik relevant für die Beurteilung seiner Theorie des ungleichen Tauschs bzw. Werttransfers auf dem Weltmarkt und der sich daraus ergebenden Momente einer Theorie der Unterentwicklung.

\section{Zur Bildung internationaler Werte}

Wie im weiteren zu zeigen sein wird, führt Mandels falsche Auffassung vom Extramehrwert, der eben nicht auf einer ,Wertübertragung“, sondern auf einer besonderen Exploitation der angewandten Arbeit beruht, in seiner Analyse des Weltmarkts (insbesondere der Weltmarktpreise und der dahinterstehenden Wertbewegung) zu einer brüchigen Ableitung der Unterentwicklung. Soweit Mandel die Unterentwicklung aus den besonderen Weltmarktbeziehungen zwischen entwickelten kapitalistischen Nationen und der Dritten Welt zu erklären versucht, steht bei ihm im Zentrum die These, daß die Entwicklung der kapitalistischen Industrienationen gleichsam durch die Unterentwicklung der Dritten Welt bezahlt sei: Der Weltmarkt wirkt seiner Ansicht nach als ein ,Siphon, der nicht nur laufend erzeugten Mehrwert, sondern auch kapitalisierten Mehrwert, d.h. Kapital aus den Halbkolonien in die Metropole transferiert.“ (20) Dabei geht Mandel (wenn dies auch nicht eindeutig aus dem Text ersichtlich ist (21)), wie auch andere neuere Theorien zur Unterentwicklung, offensichtlich davon aus, daß mit der Herausbildung eines Weltmarktzusammenhangs der kapitalistischen Produktion dieser auch die Grundlage der Wertbildung sei. Demgegenüber scheint Marx noch davon auszugehen, daß der nationale Markt sowohl die Grundlage der Wertbildung als auch einer nationalen Durchschnittsprofitrate darstelle (vgl. seine Hinweise zur Modifikation der Wertbestimmung im internationalen Austausch (22)). Nach unserer bisherigen Darstellung der Herausbildung des gesellschaftlichen Werts der Waren einer Branche müßte bei Annahme der ersten Variante zumindest nachgewiesen werden, daß sich auf dem Weltmarkt Verhältnisse hergestellt haben, die die adäquate Verteilung der internationalen gesellschaftlichen Arbeit auf die verschiedenen Produktionssphären entsprechend den auf dem Weltmarkt erscheinenden gesellschaftlichen Bedürfnissen (in Form der zahlungsfähigen Nachfrage) ermöglichen. Nur dadurch würde (umgekehrt) der Wert der Waren der Weltmarktbranche einer Nation durch einen gesellschaftlichen (hier: internationalen) Wert reguliert (23). Gleichermaßen wären da-

20) Mandel, a.a.O., S.342

21) Ebd., S.69

22) Vgl. Kapital I, 20. Kapitel, insbes. S. 584; Theorien 2, S. 101; Kapital III, 14. Kapitel, bes. S. $247 \mathrm{ff}$.

23) Hier noch einmal die Marxsche Auffassung: „Die Konkurrenz teils der Kapitalisten untereinander, teils der Käufer der Ware mit ihnen und unter sich, bewirkt hier also, daß der Wert jeder einzelnen Ware in einer besondren Produktionssphäre bestimmt ist durch die Gesamtmasse der gesellschaftlichen Arbeitszeit, die die Gesamtmasse der Waren dieser besondren Produktionssphäre erheischt, und nicht durch die individuellen Werte der einzelnen Waren oder die Arbeitszeit, die die einzelne Ware ihren besondren Produzenten und Verkäufer gekostet hat." (Theorien II, a.a.O., S.203) 
durch die erforderlichen technischen Durchschnittsbedingungen vorgeschrieben. Insofern bisher dieser Nachweis nicht in den o.a. Theorien (und bei Mandel) erbracht wurde, sollen hier im folgenden beide Varianten bei Analyse des Austauschs auf dem Weltmarkt diskutiert werden.

\subsection{Internationaler Wert und Extramehrwert}

Ist der Bezugsrahmen der Bestimmung gesellschaftlich notwendiger Arbeitszeit in einer Branche ein nationaler, wenn die gesellschaftlichen Beziehungen der Privatproduzenten sich wesentlich über den Austausch im nationalen Rahmen gestalten (d. h. der Weltmarkt noch nicht zwingende äußere Produktionsbedingung ist), so ändert sich dieser Sachverhalt, wenn die national produzierten Waren dieser Branche wesentlich für den Weltmarkt produziert werden und dieser soweit hergestellt ist, daß er wirklich die Verteilung der (internationalen) gesellschaftlichen Arbeit auf die verschiedenen Produktionssphären gemäß der auf dem Weltmarkt erscheinenden (zahlungsfähigen) Nachfrage reguliert. Insofern jetzt die national produzierten Waren einer Branche auf dem Weltmarkt mit Waren (derselben Branche) anderer Nationen mit unterschiedlicher Produktivität verglichen werden müssen, ergibt sich also die Schwierigkeit, eine einheitliche Bemessungsgrundlage für die Wertbildung der (international)gesellschaftlich notwendigen Arbeit einer Branche zu finden.Aufgrund der ungleichmäßigen und ungleichzeitigen Entwicklung der internationalen Kapitale, der historischen und politischen Besonderheiten,herrscht im Gegensatz zum nationalen Rahmen international keine durchschnittliche Produktivität und Intensität der Arbeit vor. Wir müssen vielmehr davon ausgehen, daß im internationalen Rahmen sich eine Stufenleiter und Abfolge von Produktivitäts- und Intensitätsgraden verschiedener nationaler Arbeiten insgesamt und in den Branchen hergestellt hat. Nach welchen Durchschnittsverhältnissen bemißt sich aber unter diesen Bedingungen der Wert der Waren? Marx geht im 20. Kapitel des ersten Bandes des „Kapital“ auf diese Problematik ein: ,In jedem Lande gilt eine gewisse mittlere Intensität der Arbeit, unter welcher die Arbeit bei Produktion einer Ware mehr als die gesellschaftlich notwendige Zeit verbraucht, und daher nicht als Arbeit von normaler Qualität zählt. Nur ein über den nationalen Durchschnitt sich erhebender Intensitätsgrad ändert, in einem gegebenen Lande, das Maß des Werts durch die bloße Dauer der Arbeitszeit. Anders auf dem Weltmarkt, dessen integrierende Teile die einzelnen Länder sind. Die mittlere Intensität der Arbeit wechselt von Land zu Land; sie ist hier größer, dort kleiner. Diese nationalen Durchschnitte bilden also eine Stufenleiter, deren Maßeinheit die Durchschnittseinheit der universellen Arbeit ist. Verglichen mit der weniger intensiven, produziert also die intensivere Arbeit in gleicher Zeit mehr Wert, der sich in mehr Geld ausdrückt." (24) Ohne den Vermittlungsprozeß von universeller Arbeit und branchenspezifischer. Konkurrenz auf dem Weltmarkt hier entwickeln zu wollen (25), soll festgehalten werden, daß die Durchschnittseinheit der universellen Arbeit, die als „Maßeinheit" für die Wertbestimmung der in die

24) Kapital I, S.583

25) Vgl. dazu Busch/Schöller/Seelow, a. a. O.; Neusüß3, Blanke, Altvater, Kapitalistischer Weltmarkt und Weltwährungskrise, in: Probleme des Klassenkampfs Nr. 1/1971, S. 5 ff.; Chr. Neusüß, a. a. O. Soweit in diesen Aufsätzen allerdings noch von der oben skizzierten falschen Psoition (Negicrung einer "Ubertragung von Wertbestandteilen" $z$ wischen den Branchen) ausgegangen wird, müsten dic hier gemachten überlegungen mit einbezogen werden (dies gilt insbes. für Busch/Schöller/Seelow). 
internationale Zirkulation eingehenden Waren fungiert - wenn also vom internationalen Wert ausgegangen wird - über die nationalen Durchschnitte der Arbeitsproduktivitäten und -intensitäten vermittelt sein müßte. Dabei ist der Wechselkurs insofern ein zusätzlich modifizierendes Moment, als erst durch die Austauschverhältnisse der Währungen untereinander sich jeweils die unterschiedlichen Produktivitäten und Intensitäten ausdrücken. Dadurch spiegeln die Wechselkurse einerseits die Veränderungen dieser Produktivitäten und Intensitäten wider und modifizieren andererseits - insofern in ihnen die gesamte jeweilige nationale Arbeit in ihrer Produktivität und Intensität zum Ausdruck kommt - die Austauschverhältnisse der Waren einer spezifischen Branche mit anderen Waren auf dem Weltmarkt.

Wenn es in der oben beschriebenen Weise zur Bildung internationaler Werte kommt, so ist daraus zu folgern, daß es auf dem Weltmarkt eine gesellschaftlich notwendige Arbeit gibt (für eine Branche!) und daß sich die Marktwerte und Marktpreise einer Weltmarktbranche entsprechend dem Wirken des Wertgesetzes im nationalen Rahmen - vermittelt über den Wechselkurs - herstellen. Befindet sich also ein Land durch seine außerordentliche Produktivkraft und damit überdurchschnittlich niedrigen Reproduktionskosten der Waren innerhalb einer Weltmarktbranche in der Situation, daß sich seine günstigen Produktionsbedingungen innerhalb dieser Branche noch nicht verallgemeinert haben, dann ist die Position dieses Landes gegenüber seinen Konkurrenten auf dem Weltmarkt in dieser Branche analog der Stellung eines Unternehmens mit außerordentlicher Produktivkraft (und damit eines Extramehrwertproduzenten) im nationalen Rahmen zu fassen. Der „nationale Wert" der Waren dieses Landes (d. h. seine von ihm aufgewendete nationale Arbeit zur Produktion dieser Waren - gemessen nach der Durchschnittseinheit der universellen Arbeit) liegt dann unter dem gesellschaftlichen Wert der jeweiligen Weltmarktbranche. Dieses nationale Kapital wird daher solange in dieser Weltmarktbranche einen Surplusprofit erzielen, als es sich gegenüber den internationalen Konkurrenten innerhalb dieser Branche in der besonderen Position eines Extramehrwertproduzenten befindet bzw. solange die Reproduktionskosten der Waren der anderen Länder nicht auf die des begünstigten Landes herabgedrückt wurden. Ebenso wird die Nation, deren Produktivität und Intensität der Arbeit im internationalen Vergleich in der betreffenden Weltmarktbranche schlechter abschneidet, ihre Waren unter ihren „nationalen“ Reproduktionskosten verkaufen müssen bzw. sie muß mehr nationale Arbeitsquanta zur Reproduktion dieser Waren aufwenden, um denselben Weltmarktpreis realisieren zu können, weil ein Teil dieser unter schlechteren Bedingungen verausgabten Arbeit nicht in den Wertbildungsprozeß eingeht, insofern sie nicht als Wert realisiert werden kann (26).

Sind diese Überlegungen richtig, d. h. kommt es in der oben dargestellten Weise zu internationalen Werten, dann sind Mandels Vorstellungen in den Punkten zurückzuweisen, in denen er annimmt, daß die Bildung internationaler Werte mit einem „Werttransfer“ verbunden ist. Die oben kritisierte Auffassung vom Extra-

26) Vgl. dazu auch Kapital III, S. 841: „Die Mehrarbeit wird schon deswegen nicht ganz realisiert, weil bei dem beständigen Größenwechsel der zur Produktion einer gegebnen Ware gesellschaftlich notwendigen Arbeit, der aus dem beständigen Wechse1 der Produktivkraft der Arbeit entspringt, ein Teil der Waren stets unter anormalen Bedingungen produziert und daher unter ihrem individuellen Wert verkauft werden muß." Und: "Die unter den günstigren Bedingungen produzierte Ware enthält weniger Arbeitszeit als die unter ungünstigren produzierte, verkauft sich aber zum selben Preis, hat denselben Wert, als ob sie dicselbe $\Lambda$ rbeitszeit, die sie nicht enthält, enthielte " (Thentien II, S. 204) Unter unseren Voraussetzungen (internationale Werte) gelten daher dieselben Verhältnisse! 
mehrwert führt somit bei ihm zur fehlerhaften Fassung des Surplusprofits einer Nation innerhalb einer Weltmarktbranche, den sie über ihre günstigen Produktionsbedingungen erzielen kann, denn a) gibt es eben nur einen internationalen Wert (dies unsere und Mandels Annahme (27)) und b) ist ja auch die Umverteilung von Wertbestandteilen zwischen den Branchen über den Prozeß der Herausbildung einer allgemeinen Durchschnittsprofitrate und damit der Herstellung von Produktionspreisen auf dem Weltmarkt von Mandel ausgeschlossen worden (28)!

\subsection{Nationaler Wert und Auswärtiger Handel}

Der hier vorgenommene Versuch, die Herausbildung internationaler Werte im realen Prozeß darzustellen, um auf dieser Grundlage zu klären, wie die Weltmarktpreise und die dahinterstehende Wertbewegung zu begreifen sind, ging von der Annahme aus, daß es im internationalen Rahmen noch zu keiner Durchschnittsprofitrate, zu keiner vollen Mobilität von Kapital und Arbeit gekommen ist und daß es dennoch internationale Werte gibt (was im übrigen durchaus anzweifelbar ist - aus den oben schon angeführten Gründen!). Geht man also von diesen Annahmen aus (wie Mandel), dann findet - wie gezeigt - keine Wertübertragung bei der Herstellung der Weltmarktpreise statt, sondern die Surplusprofite, die ein nationales Kapital auf dem Weltmarkt in einer Branche erzielen kann, sind über die besondere Produktivkraft der angewandten Arbeit in diesem Land zu erklären! Für die oben getroffene Annahme, daß es zur Bildung internationaler Werte kommt und daß der Weltmarkt und nicht der nationale Markt die Basis der Wertbildung darstellt, spricht allerdings die Tatsache, daß die nationalen Märkte weitgehend im Weltmarkt aufgegangen bzw. immer mehr Branchen als Weltmarktbranchen anzusehen sind, so daß der Weltmarkt sich den Produzenten der Weltmarktbranchen gegenüber bereits als bestimmende Größe aufgezwungen hat und die Akkumulation in diesen Branchen von der Weltmarktbewegung beherrscht wird.

Eine alternative theoretische Annahme ist es, die Möglichkeit einer Bildung internationaler Werte zu verneinen (infolge der o. a. Beschränkungen, die - - in einem anderen Zusammenhang - von Mandel selbst aufgezeigt werden (29)), woraus sich eine "Wertübertragung" durch den internationalen Austausch begründen ließe (in der Form des Verkaufs der Waren über oder unter nationalem Wert auf dem Weltmarkt). Mandels Position dazu läßt sich nicht genau festmachen, wie aus folgender Passage hervorgeht: „Statistisch und isoliert betrachtet, erscheint es als unwesentlich, ob man den Weltmarkt oder den nationalen Markt als wertbestimmend ansieht. Im ersten Fall findet kein Werttransfer im eigentlichen Sinne des Wortes statt, da ja auf dem Markt nicht vergütete, nicht anerkannte Arbeit, $d . h$. gesellschaftlich verschwendete Arbeit nicht wertbildend ist (eine Auffassung, die sich im geraden Gegensatz zu seiner auf Seite $94 \mathrm{f}$. aufgestellten These befindet! Der Verf.) Im zweiten Fall kann man sagen, daß die national durchaus gesellschaftlich notwendige (unter Bedingungen gesellschaftlich durchschnittlicher Arbeitsproduktivität geleistete) Arbeit international wohl geringer anerkannt wird als jene der fortgeschrittenen Länder, jedoch tatsächlich voll wertbildend ist.“"(30)

27) Vgl. etwa Mandel, a. a. O., S. 67 f.

28) Vgl. ehd.

29) Vgl. ebd., S. $324 \mathrm{ff}$.

30) Ebd., S. 331 
Nimmt man an, daß der nationale Markt die Grundlage der Wertbildung für die Weltmarktswaren dieser nationalen Kapitale ist, dann kann sich die Bildung der Weltmarktpreise in der Weise vorgestellt werden, daß die produktivere Nation aufgrund ihrer niedrigeren Reproduktionskosten der Waren bestimmte Waren über ihrem ,nationalen "Wert verkaufen kann, während die weniger produktivere Nation diese Waren nur unter ihrem ,nationalen" Wert auf dem Weltmarkt abtreten könnte. Dieser Verkauf über dem nationalen Wert wäre dann jeweils möglich, ,so oft die produktivere Nation nicht durch die Konkurrenz gezwungen wird, den Verkaufspreis ihrer Waren auf ihren Wert zu senken." (31) Diese alternative Vorstellung, daß der „,nationale“ Markt, nicht der Weltmarkt, Grundlage der Wertbestimmung ist, ist vergleichbar mit dem ungleichen Tausch zwischen Stadt und Land; ein ungleicher Tausch in dem Sinne, daß die raschere Entwicklung der Produktivität in der Stadt sich in einer ebenso raschen Senkung der Reproduktionskosten der Waren (ausgedrückt in Quantum Arbeit) niederschlägt und daß die Produktivitätsentwicklung auf dem Lande dagegen zurückbleibt (was sich in vergleichsweise höheren Reproduktionskosten der Waren dort ausdrücken müßte!). Diese ungleichmäßige Entwicklung führt in dieser Weise zum ungleichen Tausch, wenn die Produktivitäsentwicklung gegenüber dem Land ,monopolisiert" wird, d. h. wenn sich die sinkenden Reproduktionskosten der Waren der Stadt nicht in sinkenden Preisen bzw. günstigeren Austauschrelationen gegenüber dem Land ausdrücken. In solchen Übergangszeiten (32), in der der Preis der Waren nicht durch die Konkurrenz auf ihren Wert gesenkt werden kann, führt dieser Sachverhalt zu einem ungleichen Tausch von Arbeitsquanta auf Basis unterschiedlicher Produktivität und Intensität der Arbeiten. Diese Form des ungleichen Tauschs von Arbeitsquanta, die in Übergangssituationen im oben skizzierten Sinne möglich wird - etwa infolge sprunghafter Steigerung der Produktivkraft der Arbeit in bestimmten Bereichen bzw. Ländern - wäre dann auch die Form des ungleichen Tauschs zwischen Nationen unterschiedlicher Arbeitsproduktivität, wenn die Konkurrenz noch nicht dazu geführt hat, den Preis auf den Wert, d. h. auf die jeweiligen Reproduktionskosten zu senken. Solange dieser Zustand andauert, ließe sich ein Verkauf über Wert (als Nichtäquivalententausch auf dem Weltmarkt) begründen. Richtiger scheint es u. E. zu sein, diesen

31) Kapital I, S.583; vgl. auch Kapital III, S. 247 f.: , Kapitale, im auswärtigen Handel angelegt, können eine höhere Proftirate abwerfen, weil hier erstens mit Waren konkurriert wird, die von andern Ländern mit mindren Produktionsleichtigkeiten produziert werden, so daß das fortgeschrittnere Land seine Waren über ihrem Wert verkauft, obgleich wohlfeiler als die Konkurrenzländer. Sofern die Arbeit des fortgeschrittnern Landes hier als Arbeit von höherm spezifischen Gewicht verwertet wird, steigt die Profitrate, indem die Arbeit, die nicht als qualitativ höhere bezahlt, als solche verkauft wird. Dasselbe Verhältnis kann stattfïnden gegen das Land, wohin Waren gesandt und woraus Waren bezogen werden; daß dies nämlich mehr vergegenständlichte Arbeit in natura gibt, als es erhält, und daß es doch hierbei die Ware wohlfeiler erhält, als es sie selbst produzieren könnte." Vgl. dazu auch die nachfolgenden Passagen bei Marx, in denen er explizit den Vergleich mit dem Extramehrwertproduzenten angibt!

32) Vgl. dazu Kapital I, S. 474 und ebd., S. 428: „Die Maschine produziert relativen Mehrwert, nicht nur, indem sie die Arbeitsktaft direkt entwertet und dieselbe indirekt durch Verwohlfeilerung der in ihre Reproduktion eingehenden Waren verwohlfeilert, sondern auch, indem sie bei ihrer ersten sporadischen Einführung die vom Maschinenbesitzer verwandte Arbeit in potenzierte Arbeit verwandelt, den gesellschaftlichen Wert des Maschinenprodukts über seinen individuellen Wert erhöht und den Kapitalisten so befähigt, mit geringrem Wertteil des Tagesprodukts den Tageswert der Arbeitskraft zu ersetzen. Während dieser Übergangsperiode, worin der Maschinenbetrieb eine Art Monopol bleibt, sind daher die Gewinne anRerntdentlich, und der Kapitalist diese ,erste Zeit der jungen Liebe "gründlichst auszubeuten durch möglichste Verlängrung des Arbeitstags." 
ungleichen Tausch in der Weise zu fassen, daß sich unterschiedliche Arbeitsquanta tauschen, wodurch die Bedingungen der Reproduktion und Akkumulation positiv bzw. negativ beeinflußt werden. Diese Konsequenzen zu entwickeln scheint uns deshalb von Bedeutung zu sein, weil über eine solche Untersuchung des ungleichen Tauschs in seinen Wirkungen auf dem Weltmarkt die weitere Entwicklung in der Dritten Welt und in den kapitalistischen Industrienationen konkreter eingeschätzt werden kann.

\subsection{Zusammenfassung}

Diese beiden theoretischen Annahmen über die Grundlage der Wertbildung von Weltmarktswaren durch den Weltmarkt oder den nationalen Markt sind hier nur problematisiert worden, ohne daß dies in unserem Zusammenhang eindeutig hätte geklärt werden können; es sollten zunächst lediglich unklare bzw. undifferenzierte theoretische Auffassungen (wie die Mandels) anhand der verschiedenen Ableitungen kritisiert werden.

Wesentliche Vorbedingung dafür, daß der Weltmarkt zur Basis der Wertbemessung werden kann (was mit der Herausbildung internationaler Werte gleichzusetzen ist), wäre, daß der Weltmarkt schon weitgehend die Bedingung für die Produktion und Akkumulation in den meisten Branchen einer bestimmten Nation geworden ist. Der Weltmarkt müßte also schon so weit real hergestellt sein, daß dessen Bewegung die Produktion in den Branchen der nationalen Kapitale weitgehend bestimmt und sich den Produzenten in diesen Branchen als äußere Produktionsbedingung aufgezwungen hat. Ebenso müßte durch die Auflösung der nationalen Märkte und deren Aufgehen in den Weltmarkt auch schon durch diesen die Verteilung der gesellschaftlichen (internationalen) Arbeit gesteuert werden.

Aus Marx’ Äußerungen zum Weltmarkt und zum internationalen Handel kann durchaus geschlossen werden, daß er davon ausgeht, daß der nationale Markt die Grundlage der Wertbildung für die Waren im auswärtigen Handel ist. Sofern diese Interpretation zutrifft, dürfte sie Ausdruck des damaligen Entwicklungsgrades des Weltmarkts sein, auf dem die nationalen Kapitale noch nicht in dem Maße durch die Bewegung des Weltmarkts bestimmt waren, wie dies heute wohl der Fall ist. Der Grad der Exportabhängigkeit und wechselseitigen Kapitalverflechtung, die Abhängigkeit der nationalen Akkumulationsentwicklung von der Weltmarktkonjunktur und der Stellung des nationalen Kapitals auf dem Weltmarkt sprechen heute wohl zumindest bezogen auf die entwickelten kapitalistischen Nationen - eher für die Annahme, daß der Weltmarkt bereits Grundlage einer internationalen Wertbildung geworden ist und daß sich diese internationalen Werte (mit allen benennbaren Einschränkungen) in der oben dargestellten Weise herausbilden.

Es ist allerdings denkbar, daß sich die historische Konstellation und damit die Basis der Wertbildung verändern kann; d. h. daß in Phasen des Zerfalls des Weltmarkts die Wertbildung wieder nur im nationalen Rahmen ihre Grundlage hat. Ebenso kann in Prosperitätsphasen - also in Phasen zunehmender internationaler Kapital- und Warenströme und wachsender Kapitalverflechtung - der Weltmarkt erneut in seiner Relevanz für die Wertbestimmung entstehen. Dieses genauer festzumachen muß allerdings einer historischen Untersuchung überlassen bleiben. 


\section{Wesentliche Ursachen für den begrenzten Akkumulationsprozeß in der Dritten Welt (auf Basis der bisherigen Überlegungen)}

Die oben angedeuteten Formen des ungleichen Tauschs und der Preisbildung auf dem Weltmarkt sollen mun in ihren Wirkungen auf den Akkumulationsprozeß des Kapitals in der Dritten Welt untersucht werden. Im weiteren - und im Zusammenhang damit - sollen jene Faktoren benannt werden, die diesen Akkumulationsprozeß begrenzen bzw. hemmen. Dabei geht es uns nicht mehr in erster Linie um eine Auseinandersetzung mit den Thesen Mandels (hier: zur Unterentwicklung der Dritten Welt); es sollen vielmehr aus der oben geführten Auseinandersetzung Konsequenzen für die Einschätzung des Akkumulationsprozesses in der Dritten Welt angedeutet werden. (33)

\subsection{Konsequenzen des ungleichen Tauschs für den Akkumulationsprozeß in der Dritten Welt}

Um die Auswirkungen des ungleichen Tauschs, der - wie ausgeführt - seine Ursachen in der verschiedenen Produktivität und Intensität der Arbeit in verschiedenen Nationen hat, konkreter fassen zu können, ist es notwendig, die besondere Struktur der internationalen Arbeitsteilung vorweg kurz zu skizzieren. Mit der Entwicklung des Kapitalismus in Westeuropa bildete sich schrittweise eine bestimmte Produktionsstruktur des Weltmarkts heraus, die nicht zuletzt selbst wiederum eine Vorbedingung der weiteren Entwicklung des Kapitals in Westeuropa war. Innerhalb dieser Produktionsstruktur nimmt die Dritte Welt nur im bedingten Maße in den Branchen der verarbeitenden Industrie am Weltmarkt teil, ihre Produktion ist - soweit sie auf dem Weltmarkt angeboten wird - weitgehend auf die von Rohstoffen (zirkulierendes Kapital) und Konsumtionsmittel agrarischer Natur beschränkt. Auf diese extrem arbeitsteilige Produktionsstruktur, die zugleich auch die Grundlage der Nachfragestruktur auf dem Weltmarkt darstellt, sind nun die bisherigen Überlegungen zum ungleichen Tausch zu beziehen.

Wird zunächst ausgeschlossen, daß unter den bestehenden Bedingungen auf dem Weltmarkt die Monopolisierung von besonderer Produktivkraft (wie dies etwa im 19. Jahrhundert seitens Englands gegenüber seinen Kolonien der Fall gewesen ist - vgl. dazu auch die weiteren Ausführungen) möglich ist, dann können in den internationalen Werten und Austauschrelationen Produktivitätsentwicklungen zum Ausdruck kommen (gemäß der Veränderung der relativen Austauschverhältnisse einer Ware gegenüber anderen Waren, wenn die Produktivität bei der Produktion der einen oder bei der der anderen steigt). Auf das Phänomen der terms of trade gewendet, ließe sich etwa die Preisschere zwischen Rohstoffen und Fertigwaren (wir behalten hier zunächst die undifferenzierten Ausdrücke der bürgerlichen Statistik bei) in zweifacher Hinsicht erklären (ohne daß hier bereits empirische Belege angeführt werden könnten):

a) Die Produktivkraft im Rohstoffbereich wächst schneller als in der verarbeitenden Industrie. Diese Entwicklung der Produktivkraft muß zu erhöhtem Produkten-

33) Vgl. dazu auch die redaktionelle Vorbenerkung zum Aufsatz L. I:. Cardosos in diesem Heft! 
quantum führen, was ein Moment für die Tendenzen der latenten Überproduktion in den Rohstoffbranchen sein dürfte. Die extrem arbeitsteilige Produktionsstruktur des Weltmarkts bedingt dagegen eine entsprechende gesellschaftliche Nachfrage; dies führt dazu, daß angesichts der Produktivitätsentwicklung das Angebot aus den Primärgüterbranchen periodisch auf dem Weltmarkt zunimmt, ohne daß die Nachfrage in gleicher Weise Schritt gehalten hätte (was wiederum die Abhängigkeit der rohstoffproduzierenden Länder von der flukturierenden Kapitalbildung in den entwickelten kapitalistischen Ländern aufzeigt. Das Sinken der Reproduktionskosten müßte demnach dazu führen, daß angesichts der Konkurrenz der Länder der Dritten Welt untereinander in diesen Branchen die Preise fallen müßßten. Die Struktur der Weltarbeitsteilung, die in der Dritten Welt sich als Rohstoffproduktion für den Weltmarkt ausdrückt, dürfte darüberhinaus die Ursache dafür sein, daß die Kapitale in diesen Branchen in der Dritten Welt nicht ohne weiteres in andere Branchen der verarbeitenden Industrie abwandern können, da sie dort sofort auf die Konkurrenz der produktiveren Kapitale der entwickelten Industrienationen treffen würden. Es kann daher bei dieser theoretischen Alternative angenommen werden, daß die Weltarbeitsteilung und die damit zusammenhängende Nachfragestruktur die Länder der Dritten Welt latent oder periodisch in ungünstige Austauschverhältnisse mit den kapitalistischen Industrienationen zwingt.

b) Eine andere Erklärungsmöglichkeit wäre die, daß sich die Konkurrenz der rohstoffproduzierenden Länder untereinander so stark entwickelt hat, daß sie gezwungen sind, ihre Rohstoffe unter deren Reproduktionskosten zu verkaufen (d. h. auf Teile des Profits zu verzichten!), um zumindest die notwendigsten Industriegüter aus den entwickelten Industrienationen im Austausch beziehen zu können. Auch hierdurch entwickeln sich die Austauschverhältnisse zu üngunsten der Dritten Welt.

Wird gegenüber den bisher diskutierten Fällen eine Monopolisierung von besonderer Produktivkraft in den Industrieländern für möglich gehalten, so sollen jetzt auf der Grundlage der oben entwickelten zwei theoretisch möglichen Alternativen zur Basis der Wertbildung (nationaler Markt oder Weltmarkt (34)) folgende Einwände formuliert werden:

1. Die Übergangsperioden, in den sich die Reproduktionskosten der mit ausnahmsweiser Produktivkraft produzierten Waren noch nicht verallgemeinert haben (so daß über den eigenen Reproduktionskosten verkauft werden kann) sind zeitlich begrenzt und auf Dauer nicht denkbar. Über den Zwang der Konkurrenz werden diese Übergangsperioden wie auch die Position des Extramehrwertproduzenten abgebaut und ausgeglichen. Denn unter den heutigen Weltmarktsbedingungen ist es kaum denkbar, daß eine Nation in einer Branche ein Monopol gegenüber der Nachfrage auf Dauer einrichten könnte. Andere Industrienationen würden in dieselbe Branche einsteigen bzw. die Länder der Dritten Welt würden bei anderen Industrienationen einkaufen. Solche Übergangsperioden sind deshalb als dauerhafte Erscheinungen auszuschließen. Die Konkurrenz zwingt die nationalen Produzenten, die Preise auf die Werte zu senken. Dies findet bei beiden o. a. Annahmen der Grundlage der Wertbildung statt.

2. Wenn solche Übergangsperiode in einer Branche oder durch eine Nation insge-

Vgl. den 2. Abschnitt des vorliegenden Aufsatzes! 
samt nicht möglich sein kann, so könnte es doch sein, daß Ubergangsperioden in wechselnder Form permanent bestehen. Es wäre also denkbar, daß bestimmte Industrienationen oder etwa multinationale Konzerne beständig in diesem und/ oder jenem Industriezweig durch weiterentwickelte Technologie eine Übergangsperiode der Anwendung ausnahmsweiser Produktivkraft halten könnten. Dieser Fall wäre denkbar, jedoch ist er empirisch nachzuweisen.

3. Ein dritter Fall einer Monopolisierung kann dann angenommen werden, wenn die Industrieländer ein gemeinschaftliches Monopol gegenüber der Nachfrage aus den Ländern der Dritten Welt aufzubauen und zu halten versuchten und auf diese Weise gegenüber der Dritten Welt die Angleichung der Preise an die Werte verhinderten. Dieser Fall setzt jedoch voraus, daß die Waren der entwickelten Industrienationen nicht in der Dritten Welt massenhaft produziert werden könnten (was faktisch wohl zutrifft; dies vorhandene Produktionsmonopol der kapitalistischen Industrienationen auf die Produktion von Industriewaren ermöglichte ihnen zwar ein gemeinschaftliches Monopol gegenüber der Nachfrage der Dritten Welt, das Aufrechterhalten eines solchen Monopols scheint uns aber auf Dauer nicht möglich zu sein aufgrund der starken Konkurrenz der Industrienationen untereinander. Denn diese Entwicklung wird wesentlich bestimmt von den Phasen der Expansion und Kontraktion auf dem Weltmarkt insgesamt und ist nicht unabhängig davon zu denken!). Ebenso könnte dagegen die Dritte Welt ein gemeinschaftliches Monopol gegenüber den Industrienationen errichten. Auch diese Möglichkeit ist kaum realisierbar aufgrund der o. a. Konkurrenz der Länder der Dritten Welt untereinander. (Eher vorzustellen sind gemeinschaftliche Monopole gegenüber der Nachfrage nach wenigen, ganz bestimmten Gebrauchswerten, die aufgrund geographischer oder klimatischer Besonderheiten eine Sonderstellung einnehmen - wie etwa Erdöl, bestimmte Edelmetalle, tropische Produkte etc. Jedoch sind wohl derzeit auch diese Möglichkeiten bei der gegebenen Machtund Abhängigkeitsstruktur auszuschließen.)

Wenn sich auch die Phänomene der sich verschlechternden Austauschverhältnisse der Dritten Welt gegenüber den kapitalistischen Industrienationen (wie sie sich anhand der empirischen Entwicklung auch nachweisen lassen und wie sie notwendigerweise aus der Produktions- und Nachfragestruktur auf dem Weltmarkt hervorgehen) auf den ungleichen Tausch nationaler Arbeitsquanta auf Basis der unterschiedlichen Entwicklung der Produktivkraft der Arbeit zwischen der verschiedenen Nationengruppen (35) zurückführen lassen, so muß aber gerade versucht werden, die Wirkung dieser Weltmarktbeziehungen auf den Akkumulationsprozeß in der Dritten Welt konkret zu entfalten.

Nachstehend werden einige Möglichkeiten aufgezeigt; wie eine solche Vermittlung zu leisten ist. Auf diese Weise soll im einzelnen untersucht werden, wie durch die Austauschbeziehungen von der stofflichen und von der Wertseite her die Reproduktion der Klassen und die Akkumulation des Kapitals bedingt sind.

Anhand des beigefügten Schaubildes sollen die verschiedenen Möglichkeiten diskutiert werden, in welcher Weise die Austauschbeziehungen zwischen den Ländern der Dritten Welt und den Industrienationen sich auf die Reproduktion und Erweiterung des Kapitals (hier: konstantes Kapital $-c+\Delta c-$ ), die Konsumtionsverhăltnisse und das Reproduktionsniveau der Arbeiterklasse (v) und auf die Be-

Vgl. die oben entwickelten Fälle! 
standteile der Revenueverzehrer $\left(\Delta \mathrm{m}_{2}\right)$ auswirken. (36)

Die Auswirkungen der oben entwickelten Veränderungen in den Austauschverhältnissen in den Industrieländern wird die sein, daß sich der c-Teil des angewandten Kapitals verbilligt; ebenso werden die Reproduktionskosten der Arbeit in den Industrienationen gesenkt, wenn die importierten Waren agrarischer Natur wesentlich zum Bestandteil der Konsumtion der Arbeiterklasse gehören. Repräsentieren diese Waren jedoch hauptsächlich Waren, die in den Konsum der Kapitalistenklasse und der unproduktiven Schichten eingehen, dann wird sich deren Konsumtionsfond (gleichbleibender Lebensstandard vorausgesetzt) verbilligen; ein Teil der Revenue kann somit zusätzlich akkumuliert werden, oder, der Luxuskonsum dieser Klassen kann sich erhöhen, ohne daß dadurch die Masse akkumulierbaren Mehrwerts beschränkt würde! Über diese Wirkung der Austauschverhältnisse auf dem Weltmarkt wird sich daher die Profitrate der in den Industrienationen angelegten Kapitale erhöhen, insofern sich die Auslagen für c und v verringern bzw. sich die Anwendung des Kapitals verbilligt und die Mehrwertrate sich erhöht. Demgegenüber wird sich unter den diskutierten Voraussetzungen bei den in der Dritten Welt angelegten Kapitalen der für c verauslagte Kapitalteil erhöhen. Bilden die importierten Fertigwaren Teil der Lebenshaltung der Arbeiterklasse der Dritten Welt, so werden sich dadruch auch die Reproduktionskosten der Ware Arbeitskraft erhöhen. Stellen die importierten Fertigwaren einen wesentlichen Teil der Lebenshaltung der Kapitalisten und unproduktiven Schichten dar, dann wird sich deren Lebensstandard verschlechtern, wenn die Akkumulationsrate aufrechterhalten werden soll. Insgesamt wird sich die Verschlechterung der Austauschverhältnisse negativ auf den Reproduktions- und Wachstumsprozeß der Kapitale in der Dritten Welt auswirken, da sich der für $c$ und $v$ verauslagte Kapitalwert erhöhen wird (und zwar sowohl für den Ersatz vorhandener Kapitalteile wie für die Zusatzanlage von Kapital!). Dadurch verteuert sich die einfache und erweiterte Reproduktion und die Mehrwertrate fällt; die Profitrate müßte entsprechend der Bewegung dieser beiden Momente fallen!

In diesem Sinne müßten allerdings die Austausch- und Handelsbeziehungen weiter aufgeschlüsselt werden nach der Gebrauchswertform der einzelnen Warenkapitale, auf denen sie beruhen. (Dies müßte wiederum nur die internationale Arbeitsteilung widerspiegeln!) Die Auswirkungen auf die Reproduktionskosten der Waren werden sich durch veränderte Austauschverhältnisse jeweils verändern und in deren Folge auch die Lebensi altungskosten der einzelnen Klassen, deren Verhältnis zueinander im gesellschaftlichen Zusammenhang, was sich entsprechend in politischen Interessengegensätzen äußern mag.

\subsection{Zur Rolle des begrenzten äußeren und inneren Marktes}

Die extrem arbeitsteilige Produktionsstruktur und die dadurch bedingte beschränkte gesellschaftliche Nachfrage auf dem Weltmarkt führten zu den beschriebenen Auswirkungen der Austauschverhältnisse zwischen den Industrienationen und den Ländern der Dritten Welt. Es wurde schon darauf hingewiesen, daß die Länder der Dritten Welt aufgrund dieser Nachfragestruktur kaum in Branchen der verarbeiten-

36) Im Schaubild ist nur ein wichtiger Fall eingezeichnet; anhand der weiteren Aufgliederung lassen sich aber empirisch relevante Fälle unschwer studieren. Vgl. dazu auch die Erklärungen im weiteren Text! 


\section{SCHAURTLD}

Schematische Darstellung der Virkung veränderter Austauschverhältnisse auf den Reproduktions- und WachstumsprozeB in den Industrienationen und in den Jändern der Dritten Velt

Länder der Dritten Velt Industrienationen (kap.)
I. $c+v+\Delta m_{1}+\Delta m_{2}$
I. $\mathrm{c}+\mathrm{v}+\Delta \mathrm{m}_{1}+\dot{\Delta} \mathrm{m}_{2}$
II. $+\quad+v+\Delta m_{1}+\angle m_{2}$
II. $\mathrm{c}+\mathrm{v}+L \mathrm{~m}_{1}+\angle \mathrm{m}_{2}$

Veränderung der Austauschverhältnisse zuungunsten der Länder der Dritten welt:

\begin{abstract}
Gebrauchswertform lirkung des des Warenkapitals Austauschs in die Ind. Nation. auf den Reorod.proz. in der Dw
\end{abstract}

\section{1.}

c

(Produktionsmitt. meist c-zirk. $=$ Rohstoffe)

2.

Konsumtionsm.

für die Arbeiter-

klasse in den

Industrienationen)

$$
1 \cdot
$$

(Produktionsmittel, meist c-fix; verteuert!)

\section{2.}

$\mathrm{v}$

(Konsumtionsmittel für die Arbeiterklasse in der Dritten Welt; verteuert - Mehrwertrate $i$.

d. DW sinkt!)

$3 \dot{m}_{2}$ 3 . $\Delta m_{2}$ 3. $\therefore m_{2}$

(Konsumtionsm. f. (Konsumtion f. Konsumtionsg. d. Kapitalistenkl. d. Kapitalisten f.d.Kapitalisten /unprod. Schichten /unprod.Sch.i. der Dritten Vel.t i.d. Ind. Nationen)

Erklärung: d. DW verteuert, -meist Luxuspr.) Akkum. oder Kons. beschränkt!)

Profitrate sinkt, Reproduktions - und WachstumsprozeB des Kapitals beschränkt!

$\Delta \mathrm{m}_{1}$ der Teil des Mehrwerts, der akkumuliert wird, löst sich auf in' $\Delta \mathrm{c}+\Delta \mathrm{v})$

Der Übersicht halber wurde nur ein Fall eingezeichnet
Gebrauchswertform ilirkung des des Warenkapitals Austauschs in die Dritte $W$. auf den Reproduktionsproz. in den Ind.

1. $\mathrm{c}+4 \mathrm{c}$

(Produktionsmittel, verbilligt!)

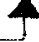

\section{2.}

(Konsumtionsfür die Arbeiter- mittel der klasse i.d. DW Arbeiterkl. -meist Fertig- i.d.Ind.N. verbilligt, Mehrwertrate steigt!)

3. $\therefore \mathrm{m}_{2}$ Konsumtionsg. f.d.Kapitalist. /unprod.Sch. verbililigt, Akk. und/oder Kons, begünstigt

Profitrate steigt, Reproduktions- $u$. Wachstumsproz. d. Kapitals befördert! 
den Industrie ausweichen können, da sie dort sofort auf die Konkurrenz der entwickelten Kapitale der Industrieländer stoßen würden. Andererseits schirmen hohe Zoll- und Handelsbarrieren die verarbeitende Industrie der entwickelten Länder gegenüber ihren Konkurrenten aus der Dritten Welt ab. Diese Beschränkung des äußeren Marktes wirkt sich also behindernd auf die Entwicklung der Produktivkraft der Arbeit in den Ländern der Dritten Welt aus. Gerade am Aufstieg des Kapitalismus in Westeuropa läßt sich dagegen verfolgen, daß die Entfaltung von Produktionspotenzen verbunden war mit einer beständigen Ausweitung der Absatzgebiete, der Herstellung des Weltmarkts. In diesem Sinne ist der Weltmarkt auch als Voraussetzung der Steigerung der Produktivkraft der Arbeit zu verstehen, insofern er erst eine Aufnahme der Produktion in großem Umfange, die Massenproduktion, die Konzentration und Zentralisation von Kapital und damit erst die Freisetzung aller gesellschaftlichen Produktionspotenzen ermöglicht.

Sind die Zoll. und Handelsbarrieren in diesem Sinne durchaus Hemmnisse der weiteren Entwicklung der Produktivkräfte und damit der Akkumulation des Kapitals, so muß hier schon festgehalten werden, daß die Enge der Märkte nicht als primäre Ursache für die zögernde Kapitalakkumulation in der Dritten Welt anzusehen ist. Diese Argumentation, über den begrenzten Markt die schleppende Industrialisierung zu erklären, findet sich aber in nahezu allen Abhandlungen und Auseinandersetzungen zum Problem der Unterentwicklung. (37) Auch Mandel argumentiert in ähnlicher Weise: „Indem billige Arbeitskraft unter gesellschaftlichen Bedingungen aufgefunden und reproduziert wird, unter denen die Arbeitsteilung einerseits noch nicht weit verbreitet ist, andererseits die Senkung des Wertes der Arbeitskraft auf die physischen Reproduktionskosten jegliche Erweiterung der Bedürfnisse und daher jegliche Ausdehnung des inneren Marktes verhindert, entsteht eine durch das Kapital selbst hervorgerufene und nicht zu überwindende Schranke für seine eigene Expansion.“ (38) Oder an einer anderen Stelle: „Der in den abhängigen, halbkolonialen Ländern geringere Preis der Arbeitskraft erlaubt wohl eine höhere durchschnittliche Profitrate - die letzten Endes erklärt, warum ausländisches Kapital überhaupt in diese Länder einfließt - schafft jedoch gleichzeitig Schranken für die weitere Akkumulation des Kapitals, weil der Ausdehnung des inneren Marktes durch die niedrigen Reallöhne und die geringen Bedürfnisse der Arbeiter enge Grenzen gezogen sind.“ (39) Die von Mandel hier angeführten Faktoren, aufgrund derer der Absatzmarkt begrenzt sein soll, mögen jeweils nachweisbar und für eine konkrete Analyse der Unterentwicklung von Bedeutung sein; jedoch ist dieser Argumentation generell entgegenzuhalten:

1. schlösse dann der niedrige Preis der Arbeitskraft eine hohe Mehrwertrate und damit ein hohes akkumulationsfähiges Mehrprodukt ein; insofern ist auch der niedrige Preis der Arbeitskraft immer schon Voraussetzung der beschleunigten Industrialisierung gewesen. Andererseits ist - gerade weil der Stand der Entwicklung der Produktivkraft der Arbeit in der Dritten Welt so niedrig ist - zu bezweifeln, daß - verglichen mit den produktiveren Industrienationen - der niedrige Preis der Arbeitskraft in einer höheren Mehrwertrate seinen Ausdruck findet, da die Reproduktionskosten der Arbeitskraft vergleichsweise gleichwohl höher sein

37) Zu den verschiedenen Begründungszusammenhängen in diesen Theorien und deren Fehlerhaftigkeit vgl. das redaktionelle Vorwort 7.u F. F. Cardoso in diesem Heft.

38) Mandel, a. a. O., S. 79.

39) Ebd., S. 64. 
können. Die (angenommene) hohe Profitrate kann ebenso in der niedrigen organischen Zusammensetzung seine Ursache haben.

2. kann der immanente Gegensatz bei Mandel (und bei den anderen Theoretikern), der darin besteht, einerseits eine hohe Profitrate (weil hohe Mehrwertrate) und damit die Möglichkeit der Kapitalbildung und Industrialisierung, andererseits aufgrund der niedrigen Löhne und des damit begrenzten inneren Marktes die Unmöglichkeit der Akkumulation des Kapitals in diesen Ländern zu postulieren, nur dadurch entstehen, daß in diesen Theorien die Kapitalisierung eines Teils des Mehrprodukts (der sich überhaupt nicht in Lohn und Revenue auflöst) nicht gesehen wird. Gerade diese Kapitalisierung (was die erweiterteNachfrage nach Warenkapital einschließt) erweitert aber den inneren Markt, ohne daß die individuelle Konsumtion dabei eine Rolle spielen mag!

Die Schwierigkeiten für die Länder der Dritten Welt zu akkumulieren, die neben den schon benannten Auswirkungen des ungleichen Tausches in den hier angeführten inneren und äußeren Beschränkungen (die aber nicht unüberwindbar sind) zu se'hen sind, führen insgesamt dazu, daß die Kapitalakkumulation nur zögernd in Gang kommt und deshalb die Arbeitsteilung und die Entwicklung der Produktivkraft der Arbeit nur schleppend weitergetrieben werden kann (weshalb auch die vorkapitalistischen Produktionsweisen nur langsam aufgelöst werden und sich relativ lange innerhalb der kapitalistischen Warenzirkulation behaupten können (40)). Die schleppende Industrialisierung über die Arbeitslosigkeit, den begrenzten inneren Markt usw. zu erklären, heißt an den primären Ursachen des begrenzten Prozesses der Entwicklung der Produktivkraft der Arbeit und der Kapitalakkumulation vorbeizugehen, die die Ausdehnung des inneren und äußeren Marktes selbst wiederum begrenzen.

Die Unterentwicklung, unter die die verschiedensten Phänomene in den aktuellen Theorien subsumiert werden, ist - dies ist als Ergebnis der vorstehenden Überlegungen insgesamt festzuhalten - nur in der Weise analysierbar, daß die Gesetzmässigkeiten und Strukturen des kapitalistischen Weltmarkts sowie die spezifischen ökonomischen und gesellschaftlichen Bedingungen in den Ländern der Dritten Welt unter dem Gesichtspunkt der Möglichkeit und der Form der Akkumulation des Kapitals und der darin gesetzten Gegensätze und Widersprüche betrachtet werden. In diesem Sinne sind die oben angeführten Thesen zunächst als negative Abgrenzung zu Theorien zu verstehen, die diese Möglichkeit überhaupt negieren oder doch nur im äußerst begrenzten Rahmen zu sehen vermögen. Eine Antwort auf die weiterführenden Fragen, welche Klassenstrukturen, gesellschaftliche Kräfteverhältnisse und Widersprüche in der Dritten Welt durch den Entwicklungsprozeß des Kapitals hervorgetrieben werden, in welchen besonderen Formen sich die Produktivkräfte entwickeln werden, welche Chancen die Kapitalakkumulation und damit die Kapitalherrschaft hat, welche Rolle der Staatsapparat dabei spielt, erfordert zunächst, die Tendenzen auf dem Weltmarkt in ihrem Zusammenhang mit der inneren Struk-

40) Die immense versteckte und offene Arbeitslosigkeit ist daher eher der Ausdruck einer (bei in Zersetzung begriffenen vorkapitalistischen Produktionsweisen) nur schleppenden Kapitalakkumulation und nicht Resultat eines voll entfalteten industriellen Kapitals, das über Konzentrations- und Zentralisationsprozesse, Kapitalisierung von Produktionsbedingungen etc. massenhaft Arbeitskräfte freigesetzt hat und permanent Arbeitskraft aus dem Produktionsprozcß hinauswirft und wicder cinsaugt. Dieses Stadium gilt es erst in den Ländern der Dritten Welt zu erreichen, was durchaus nicht trostreich für die $\mathrm{Be}$ troffenen ist. 
tur der Länder der Dritten Welt selbst zu untersuchen. Denn die Chancen der Kapitalakkumulation in der Dritten Welt werden weitgehend durch die Entwicklungstendenzen in und zwischen den Industriestaaten bestimmt; wie andererseits die Kapitalakkumulation in den Industrieländern und damit die Herrschaft des Kapitals überhaupt von der Möglichkeit und dem Ausmaß der Kapitalakkumulation in der Dritten Welt betroffen sein dürfte.

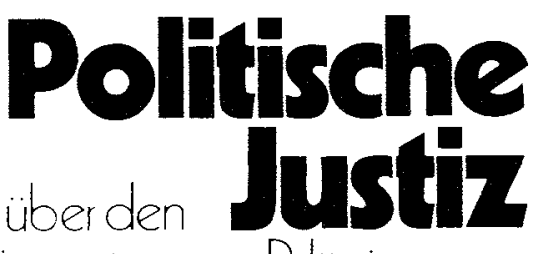

Dokumentation über den Ausverisungsterror an Palastinensern

Herausgeber: Rechtsanwaltbüro Groenewold, Degenhardt, Reinhard

Aus der Dokumentation ergibt sich, daß das Ausländergesetz kein Gesetz ist, das dem Recht zuzuordnen ist, sondern daß durch seine Plastizităt Aufenthalt und Existenz je nach Interessen der herrschenden Klasse zur freien Verfügbarkeit der Ausländerbehorden hält. Die Gerichte haben ihre Funktion, die Ausweisungen an den Grund - und Menschenrechten zu messen und zumindestens einstweilen zu verhindern, nicht wahrgenommen. 176 S. $6,-\mathrm{DM}$

Einzelversand durch:

MANIFEST -Buchversand

2 Hamburg 13, Schlüterstr. 79

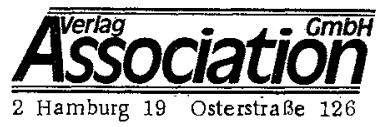

\section{J. Ghmelnizkaja}

Der

Uiffidanteth:

IIIOIOPO-

kapitailismus

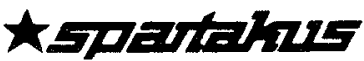

2 Hamburg 13. Postfach 117 
Charles Betteheim

\section{Theorie und Praxis sozialistischer Planung}

trikonttheOre tRIKONT-vERLAG 8 München 80 , Josefsburgstr. 16

EINFUHRUNG: Detinition des Plans/Direktiven/Konjunkturprognostik/Produktion und gesellschaftlicher Bedarf/Planung der Produktion/Planung der Verteilung

ZENTRALISIERTE ENTSCHEIDUNG ODER DEZENTRALISIERTE ENTSCHEIDUNG: Konzeption der dezentralisierten Entscheidung und ihre Kritik/Grenzen der Zentralisierung/Sozialstruktur und wirtschaftliche Berichtigungen

DIE ENTSCHEIDUNG UND DAS WERTPROBLEM: Die Entscheidung im Bereich der Produktion/Arbeitszeit als planwirtschaftliche Rechnungseinheit

WIRTSCHAFTLICHE ENTSCHEIDUNG UND BEDARFSBESTIMMUNG: Quantitative Bestimmung des Konsumtionsbedaris/ Qualitative Bestimmung des Produktionsbedarfs/Wirtschaftliche Entscheidung in einer Planwirtschaft

GELD UND RECHNUNG IN GELD: Geld als Wertmaßstab/Zirkulations- und Zahlungsmittel/Vergütungsmittel/Spar- und Akkumulationsmittel

PREISE UND GEWINNE: Wert/Herstellungspreis/Großhandelspreis/Einzelhandelspreis/Gewinn

BESTIMMUNG DER GUNSTIGSTEN TECHNIK: Rentabilität/Arbeitszeiteinsparung/Bewegung der Arbeitsproduktivität/Dauer der Anlauizeit/Direkte und indirekte Investitionen

DAS PROBLEM DES OKONOMISCHEN GLEICHGEWICHTS: Optimale Verwendung/Gleichgewicht zwischen Produktion und gesellschaftlichem Bedarf/Optimale Verwendung der Arbeitskraft

GLEICHGEWICHT ZWISCHEN PRODUKTION UND KONSUMTION: Problem des stationären Zustands 


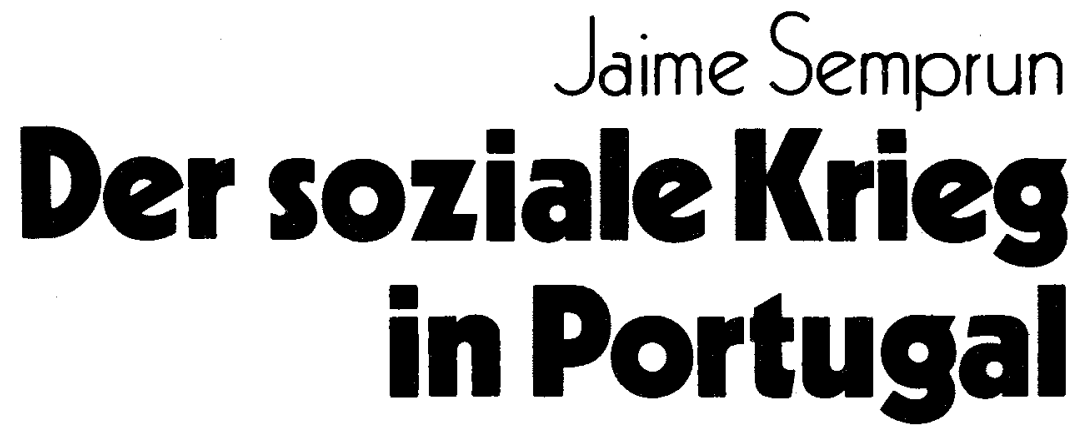

Hier endlich ein respektloses, intelligentes und explosives Buch. Wie die Texte der Situationisten, von denen es offenbar inspiriert ist, wird es manche vor den Kopf stoßen: er ist kurz und intensiv, brillant geschrieben (was so viele linke Bücher bei uns so sehr vermissen lassen!) und ohne jegliche Höflichkeit. Der Autor macht auch nicht halt vor dem Mythos der Linksradikalen, die nichts anderes sind als die letzte Zuflucht der herrschenden Ideologie.

Er entdeckt im Kampf des portugiesischen Proletariats eine neue alte Qualität: die der Autonomie. Die radikalsten und bewußtesten Arbeiter haben alle Parteien und Sekten mit ihren Stellvertreter- und Avantgardeansprüchen hinter sich gelassen.

„Was seit September die besessenen Anrufungen der Vergangenheit und das angstvolle Schweigen über die Gegenwart nur schlecht verbergen konnten, das sind Geburt und Fortschritte einer modernen Revolution, ohne Fahne und Ideologie". Er zieht eine Linie von der Pariser Commune über die Soviets von 1905 und 1917, Kronstadt 1921, die spanischen Kollektivitäten 1937, den Räten von Budapest 1956 bis zu den Komitees von Danzig 1970, von LIP bis LISNAVE. DM 5.50

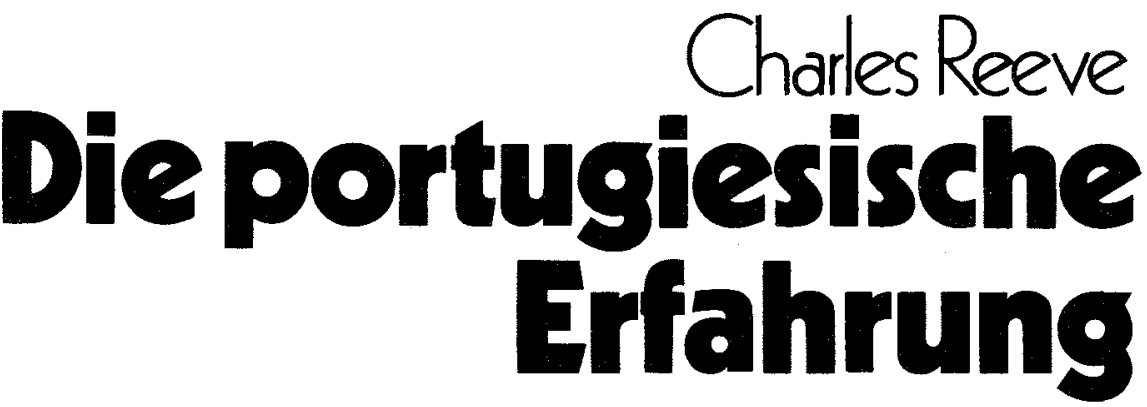

Der Autor von „Der Papiertiger" analysiert die letzten Entwicklungen der portugiesischen Revolution und kritisiert vor allem die militaristische Konzeption der Linksradikalen. ca. 40 S. ca. DM 4.00 


\section{Rita di Leo}

\section{Die Arbeiter und das sowjetische System Die Entwicklung von Klassenstrukturen und Klassenherrschaft in der UdSSR}

\section{Iflkont theOrie TRIKONT-VERLAG - 8 München 80, Josefsburgstr. 16}

Eine Bestandsaufnahme der Geschichte der Sowjetunion - unter der Fragestellung: wie hat sich die Lage der Arbeiterklasse in der Sowjetunion im letzten halben Jahrhundert entwickelt?

Eine solche Fragestellung ist nur sinnvoll, wenn der Kernpunkt ,sozialistischer" Ideologie durchbrochen wird, nämlich die Annahme, durch die Aufhebung privater Verfigungsgewalt über die Produktionsmittel und die Beseitigung der Anarchie des Marktes sei bereits eine Alternative zum Kapitalismus geschaffen. Die Fragestellung geht vielmehr von dem aus, was ein Blick in die Fabrikrealität der UdSSR erbringt: hier setzt sich die alte Produktionsweise fort - der Gebrauchswert der lebendigen Arbeit bleibt den prinzipiell gleichen Bedingungen unterworfen wie im Kapitalismus.

Dargestellt wird die Entwicklung der Lage der Arbeiterklasse anhand der Funktion der NEP-Politik, des Stachanovismus, der Jafirespläne und der Wirtschaftsreformen der nachstalinschen Ära - immer unter der Fragestellung: Wie hat sich das Verhälthis von Kapital und Arbeit verändert? Die Ursachen für die Entwicklung sieht die Autorin nicht, wie so viele, in einer einfachen „Burrokratisierung" und Entfernung der Partei von den Massen. Diese Entfremdung hat wieder ihre Ursachen: den Versuch, eine sozialistische Gesellschaft schematisch am Modell der Marx'schen Reproduktionsschemata zu orientieren; diese aber waren ein Modell des jugendlichen Kapitalismus, nicht einer sozialistischen Alternative.

Die zweite theoretische Fehlentscheidung lag darin, daß die Gultigkeit des Wertgesetzes fü die sozialistische Wirtschaft iber 20 Jahre lang völlig geleugnet wurde. Daraus ergab sich eine bestimmte Art der Plandurchführung, die jede reale Bewertung und Erfolgsrechnung, jedes Kostenbewußtsein verhinderte.

Die Leugnung des Wertgesetzes fuhrte außerdem dazu, das der Gegensatz zwischen Staat und Arbeiterklasse nicht auf den Begriff gebracht werden konnte. 
C. Chatterley u.a.

Feminismus oder Erotik

Die Entdeckungen der Venus

und die Abenteuer des

Phallus innerhalb der Bewe-

gung des Kommunismus

Flugschrift No. 20,60 $\$ / 5,00$

Schriften zur Auflehnung gegen

die Herrschaft von Kapital und

Ideologie über den Korper
Erich Mühsam

Von Eisner bis Leviné. Die Entstehung und Niederlage der baverischen Rätereoublik. Ein Bericht.

Fiugschrift No. 17, 90 S/6.00 Revolutionare Begeisterung, Dilletantismus und weiber Terror in Bayern.

\section{Neuerscheinungen}

Peter Paul Zahl

Die Barbaren kommen

Lyrik und Prosa

Flugschrift No. $15,158 \mathrm{~S} /$

8.00 DM

in einer poetischen Sprache setzt Zahl eine Collage der politischen und sozialen Realität unserer Tage zusammen.

\section{Zeitschrift Revolte}

Organ der Subrealisten

No. 15/16

Editorial + Keiner soll entkommen, Notizen über zeitgenössische Erscheinungen + Welt der Bilder + Ich wollte das Rot von Techni-Color + Vorläufige Notizen zum Leben der Studenten + Definitionen + Dokumented. Subrealisten + Statistische Mitteilungen u.a.

\section{Doppelnummer 6.00 DM}

Bob Potter

Vietnam Superstar - Sieg für wen?

Flugschrift No. $12,96 \mathrm{~S} / 6.00$ Kritische Analyse der vietnamesischen Ereignisse. Der Autor liefert eine entmystifizierend e Analyse dieses Krieges der Supermächte seit 1945, indem er dessen, ,innere Geschichte" der ,weltpolitischen" gegenüberstellt.

Fordern Sie unseren Verlagskatalog an!
Heidi Schmidr

Anfälle. Tagebuchfragmente

Flugschrift No, 16, ca, $100 \mathrm{~s} /$ $7.00 \mathrm{DM}$

Der Versuch einer Fras, ihre psychischen Stimmungen , abzuschreiben", macht dieses Buch zum Diálog zwischen dem "Ich" und dem ötfentlichen "Wir".

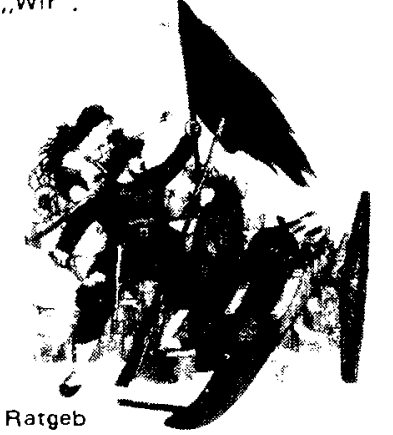

Vom wilden Streik zur generalisierten Selbstverwaltung

Flugschrift No. $11,985 / 6.00$ Zur Strategie, Taktik und Utopie des aufständischen Prolezariats.

\section{Paul Cardan}

\section{Postskript zur Neudefini-} tion der Revolution

Flugschrift No. $6,69 \mathrm{~s} / 4,80$ „Die Revolutionäre Bewegung muß damit aufhören, wie eine politische Bewegung im traditionellen Sinne zu erscheinen."
SITUATIONISTISCHE INTERNATIONALE 1958-1969

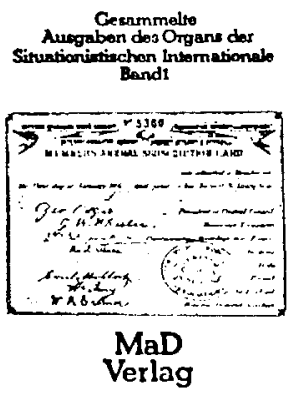

In dieser Zeitschrift spiegeln sich die Ideen und Ereignisse der letzten 20 Jahre, gleichzeitig ist sie eine der Antworten auf die aufgeworfenen Fragen unserer Epoche.

Die Situationisten haben die Theorie der unterirdischen Bewegung aufgedeckt, die das moderne Zeitalter quält und mit ihrem aufständischen Stil die Negation in einer Welt, die sich im Positivismus aufgebläht hat, neu belebt. Sie haben letztlich nicht nur gewagt zu denken, sondern vielmehr gewagt zu leben

\section{Pressestimmen:}

„Viele der Parolen, die auf den Mauern von Paris Berühmtheit erlangten, können hier in irgendeiner form gefunden werden ..." Times, Literaturbeilage.

,.Der Situationismus ist kein größeres Gespenst, das in der ind ustriellen Gesellschaft umghet, als der Kommunismus das Gespenst war, das 1848 in Europa umging." Nouvel Observateur.

"Eine zähnefletschende Rhetorik, die immer maßlos ist..." Le Monde

$329 \mathrm{~S} / 22.00 \mathrm{DM}$

Überformat im OriginalLayout

\section{"MaDVerlag

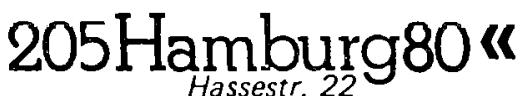

